\title{
INDUCED HOPF CORING STRUCTURES
}

\author{
Rui Miguel Saramago
}

\begin{abstract}
Hopf corings are defined in this work as coring objects in the category of algebras over a commutative ring $R$. Using the Dieudonné equivalences from [7] and [19], one can associate coring structures built from the Hopf algebra $\mathbf{F}_{p}\left[x_{0}, x_{1}, \ldots\right], p$ a prime, with Hopf ring structures with same underlying connected Hopf algebra. We have that $\mathbf{F}_{p}\left[x_{0}, x_{1}, \ldots\right]$ coring structures classify thus Hopf ring structures for a given Hopf algebra. These methods are applied to define new ring products in the Hopf algebras underlying known Hopf rings that come from connective Morava $k$-theory.
\end{abstract}

\section{Introduction}

This work follows closely the notations from [7], [4] and [18] for Hopf rings and their universal bilinear products, and [20] for Hopf corings and their universal cobilinear coproducts. The constructions of such objects are presented here in Sections 2 and 3.

Taking the Witt algebra $\mathbf{F}_{p}\left[x_{0}, x_{1}, \ldots\right]$, viewed as a Hopf algebra whose primitives are the Witt polynomials, one can obtain different Hopf corings, each coming from a specific second coproduct on $\mathbf{F}_{p}\left[x_{0}, x_{1}, \ldots\right]$.

The Dieudonné equivalence between Hopf rings and Dieudonné rings in various settings ([7], [19], [4]) permits us to relate those corings $\mathbf{F}_{p}\left[x_{0}, x_{1}, \ldots\right]$ with new Hopf rings coming from given Hopf rings. Specifically, Proposition 4.1 says that, if $H$ is a connected Hopf ring, one can get a second Hopf ring (with the same underlying Hopf algebra) for each coring structure on $\mathbf{F}_{p}\left[x_{0}, x_{1}, \ldots\right]$.

The induced Hopf ring products are then applied to obtain new Hopf ring structures arising from known objects in connective Morava $k$-theory. As examples, one gets products that come from the diagonal and $k$-shift coring structures on $\mathbf{F}_{p}\left[x_{0}, x_{1}, \ldots\right]$, but many others can be developed in a similar way.

Received February 12, 2010; Revised May 5, 2010.

2010 Mathematics Subject Classification. 16W30, 57T05, 18E10.

Key words and phrases. Hopf algebras, Hopf rings, Dieudonné modules, homotopy theory. 


\section{Connected Hopf algebras and rings and the Dieudonné equivalence}

Let $H_{1}, H_{2}$ and $K$ be Hopf algebras over a commutative ring $R$. A morphism of coalgebras

$$
\phi: H_{1} \otimes H_{2} \rightarrow K
$$

is called a bilinear map of coalgebras if we have:

(1) $\phi(x y, z)=\sum \phi\left(x, z^{(1)}\right) \phi\left(y, z^{(2)}\right)$,

(2) $\phi(x, y z)=\sum \phi\left(x^{(1)}, y\right) \phi\left(x^{(2)}, z\right)$,

(3) $\phi(x, 1)=\epsilon(x) \cdot 1$,

(4) $\phi(1, y)=\epsilon(y) \cdot 1$,

where $\epsilon$ is the counit and superscripts come from the coproduct $\psi$ (following Sweedler's notation to get $\psi(x)=\sum x^{(1)} \otimes x^{(2)}$ for any $\left.x\right)$.

Hopf algebras have universal bilinear products $\otimes([7])$. For each pair of Hopf algebras $H_{1}$ and $H_{2}$, this is defined as the unique Hopf algebra $H_{1} \otimes H_{2}$ together with a bilinear map (of coalgebras)

$$
\gamma: H_{1} \otimes H_{2} \rightarrow H_{1} \otimes H_{2}
$$

such that for any bilinear map $H_{1} \otimes H_{2} \rightarrow K$ there exists a unique Hopf algebra map $H_{1} \otimes H_{2} \rightarrow K$ that makes the following diagram commute:

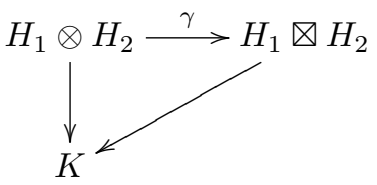

This universal bilinear product is constructed, in each case, as a quotient of the symmetric algebra $S\left(H_{1} \otimes H_{2}\right)$ on $H_{1} \otimes H_{2}$. This symmetric algebra has a coproduct given by the requirement that the inclusion $H_{1} \otimes H_{2} \rightarrow S\left(H_{1} \otimes H_{2}\right)$ be a map of coalgebras. That is, for $a \in H_{1}$ and $b \in H_{2}$, we have

$$
\psi(a \otimes b)=\sum\left(a^{(1)} \otimes a^{(2)}\right) \otimes\left(b^{(1)} \otimes b^{(2)}\right) .
$$

Consider the ideal $J$ in $S\left(H_{1} \otimes H_{2}\right)$ generated by the elements

(1) $(x y) \otimes z-\sum\left(x \otimes z^{(1)}\right) \otimes\left(y \otimes z^{(2)}\right)$,

(2) $x \otimes(y z)-\sum\left(x^{(1)} \otimes y\right) \otimes\left(x^{(2)} \otimes z\right)$,

(3) $x \otimes 1-\epsilon(x) \cdot 1$

(4) $1 \otimes y-\epsilon(y) \cdot 1$.

Proposition 2.1 ([4]). The product and coproduct on $S\left(H_{1} \otimes H_{2}\right)$ that were presented above induce in $S\left(H_{1} \otimes H_{2}\right) / J$ a structure of Hopf algebra that makes it the universal bilinear product $H_{1} \otimes H_{2}$ of $H_{1}$ and $H_{2}$.

These are the properties used in the proof of the equivalence between categories of Hopf rings and Dieudonné rings.

The construction of $H_{1} \otimes H_{2}$ permits us to give a straight definition of Hopf rings. A commutative Hopf ring over a commutative ring $R$ is a Hopf 
algebra $H$ over $R$ together with a map $\phi: H \otimes H \rightarrow H$ that is associative: $\phi(\phi \otimes 1)=\phi(1 \otimes \phi)$.

This definition is equivalent to saying there has to be a circle product $\circ$ : $H \otimes H \rightarrow H$, which is a map of coalgebras, satisfying convenient distributivity properties with respect to the algebra product ([15]), as given by the following commutative diagrams

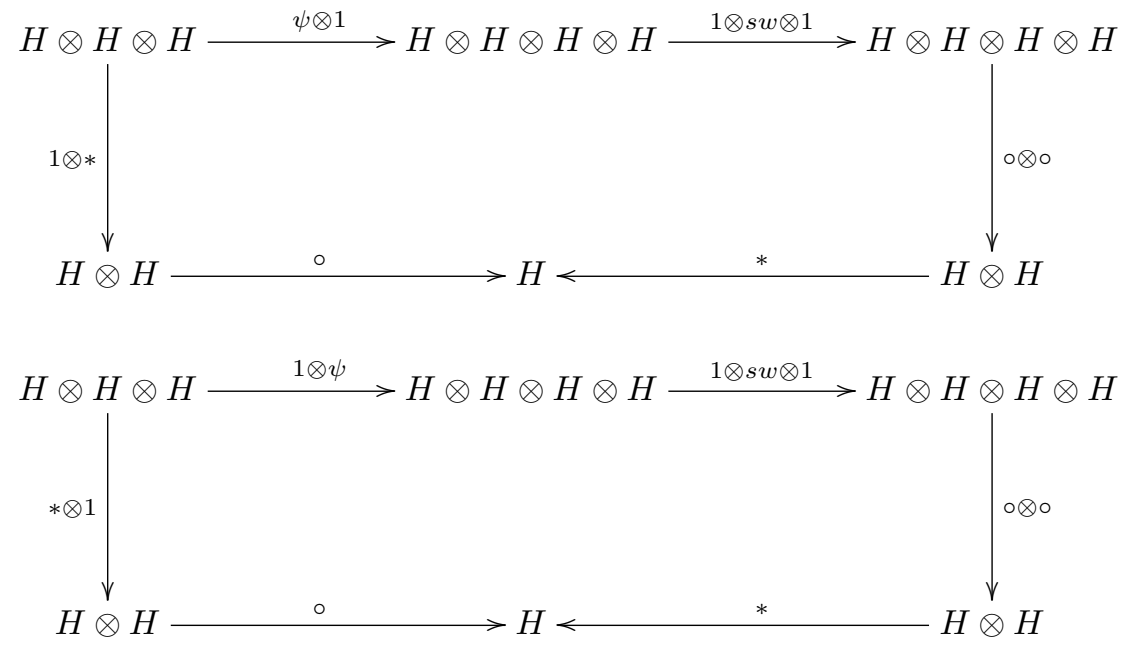

(Here, $*$ represents the algebra product and $s w$ is the switch map taking $a \otimes b$ to $b \otimes a)$.

Let $\mathcal{H} \mathcal{A}$ be the category of ungraded connected Hopf algebras over $\mathbb{F}_{p}, p$ a prime. Given a sequence of indeterminates $\left\{x_{i}\right\}$, we consider the Hopf algebras $H(n)=\mathbb{F}_{p}\left[x_{1}, \ldots, x_{n}\right]$ for $n \geq 0$. We have Hopf algebra maps $\alpha: H(n) \rightarrow$ $H(n+1)$ (given by inclusion) and $\bar{V}: H(n+1) \rightarrow H(n)$ (defined by $\bar{V}\left(x_{i}\right)=$ $x_{i-1}$ for $i>0$ and $\left.\bar{V}\left(x_{0}\right)=0\right)$. $\bar{V} \alpha$ gives the Verschiebung on each $H(n)$. We have thus a sequence

$$
\cdots \longrightarrow H(n+1) \stackrel{\bar{V}}{\longrightarrow} H(n) \stackrel{\bar{V}}{\longrightarrow} H(n-1) \longrightarrow \cdots .
$$

For each ungraded connected Hopf algebra $H$, this sequence induces a sequence of $\mathbb{F}_{p}$-modules

$\cdots \rightarrow \operatorname{Hom}_{\mathcal{H A}}(H(n-1), H) \stackrel{\hat{V}}{\rightarrow} \operatorname{Hom}_{\mathcal{H} \mathcal{A}}(H(n), H) \stackrel{\hat{V}}{\rightarrow} \operatorname{Hom}_{\mathcal{H A}}(H(n+1), H) \rightarrow \cdots$ where each $\hat{V}$ is given by composition with $\bar{V}$ on the left.

Consider now the $\mathbb{F}_{p}$-module $D H=\operatorname{colim}_{n} \operatorname{Hom}_{\mathcal{H A}}(H(n), H)$. Composing on the right with the Verschiebung $v: H \rightarrow H$ and the Frobenius $f: H \rightarrow H$ (defined as $f(x)=x^{p}$ for any $x$ ) gives maps $V: D H \rightarrow D H$ and $F: D H \rightarrow$ $D H$. We have $F V=V F=p$. Given a Hopf algebra $H \in \mathcal{H} \mathcal{A}$, we define its Dieudonné module as the module $D H=\operatorname{colim}_{n} \operatorname{Hom}_{\mathcal{H} \mathcal{A}}(H(n), H)$ together with the homomorphisms $V: D H \rightarrow D H$ and $F: D H \rightarrow D H$ given above. 
For each $n$, denote by $\varphi^{n}: \operatorname{Hom}_{\mathcal{H A}}(H(n), H) \rightarrow D H$ the morphism coming from the definition of the direct limit.

Since any $H \in \mathcal{H} \mathcal{A}$ is connected, its coargumentation filtration $\left\{F_{q} H: q \in\right.$ $\mathbf{N}\}$ exhausts it and, moreover, if we write $\psi(x)=1 \otimes x+x \otimes 1+\sum x^{\prime} \otimes x^{\prime \prime}$ for each $x \in F_{q} H$, then all the $x^{\prime}$ and $x^{\prime \prime}$ that appear in the expression are in those $F_{q^{\prime}} H$ that have $q<q^{\prime}$. Thus, the Verschiebung on such Hopf algebras is eventually zero. This carries over to $D H$, where we have that for each $x \in D H$ there must exist $n \geq 0$ such that $V^{n} x=0$.

Definition 2.2. The category $\mathcal{D} \mathcal{M}$ of ungraded connected Dieudonné modules has as objects modules $M$ over the ring $R=\mathbb{Z}_{p}[V, F] /(V F=F V=p)$ such that for each $x \in M$ there exists $n \geq 0$ with $V^{n} x=0$.

The above considerations give us a functor $D: \mathcal{H} \mathcal{A} \rightarrow \mathcal{D} \mathcal{M}$ that takes a Hopf algebra $H \in \mathcal{H} \mathcal{A}$ and produces its Dieudonné module

$$
D H=\operatorname{colim}_{n} \operatorname{Hom}_{\mathcal{H A}}(H(n), H) \text {. }
$$

Theorem $2.3([3])$. The functor $D: \mathcal{H} \mathcal{A} \rightarrow \mathcal{D} \mathcal{M}$ has a left adjoint $U: \mathcal{D} \mathcal{M} \rightarrow$ $\mathcal{H} \mathcal{A}$, and the pair $(D, U)$ forms an equivalence of categories.

One defines a bilinear map for $R$-modules $M, N$ and $L$ as a map $g: M \otimes N \rightarrow$ $L$ that satisfies:

(1) $V g(m \otimes n)=g(V m \otimes V n)$,

(2) $F g(V m \otimes n)=g(m \otimes F n)$,

(3) $F g(m \otimes V n)=g(F m \otimes n)$

for every $m \in M$ and $n \in N$ (This is a similar definition to that made for Hopf algebras).

Two Dieudonné modules $M$ and $N$ also have a universal bilinear product, given by a Dieudonné module $M \otimes N$ together with a bilinear map $M \otimes N \rightarrow$ $M \otimes N$ that is universal with respect to all bilinear maps ([7], [4]).

The following result gives the fundamental equivalence between the category of ungraded connected Hopf rings and that of connected Dieudonné rings, which are ungraded connected Dieudonné modules together with a product compatible with $V$ and $F$.

Theorem 2.4 ([7], [4]). The category $\mathcal{D} \mathcal{M}$ together with the bilinear product $\otimes$ is a monoidal category which is equivalent to the category of ungraded connected Hopf rings.

From [19], we get that a product on a connected Hopf algebra $H$ induces a product on its Dieudonné module $D H$, and the same holds in the opposite direction. This reflects the equivalence between ring structures on $H$ and $D H$ that the previous theorem declares.

Specifically, suppose one has a product on $D H$ and wants to define a product on $H$. Take a primitive $x \in H$. Pick a positive $m$ and define $\hat{x} \in$ $\operatorname{Hom}_{\mathcal{H} \mathcal{A}}(H(m), H)$ by $\hat{x}(1)=1, \hat{x}\left(\omega_{m}\right)=x$ and $\hat{x}\left(\omega_{i}\right)=0$ for $i \neq m$. $\hat{1} \in \operatorname{Hom}_{\mathcal{H A}}(H(0), H)$ is defined as $\hat{1}(a)=1$ for all $a$. Then $\varphi^{m}(\hat{x}) \circ \varphi^{0}(\hat{1})$ 
is in $D H$, so it should be $\varphi^{m}(\alpha)$ for some $\alpha \in \operatorname{Hom}_{\mathcal{H A}}(H(m), H)$. This $\varphi^{m}(\alpha)$ defines $\hat{x} \circ \hat{1}$. The product in $H$ is then given by $x \circ 1=\hat{x} \circ \hat{1}\left(\omega_{m}\right)$. Similarly, we define $1 \circ y$ for a primitive $y$. If we are working with a characteristic zero ring, this is enough (since the primitives of $H \otimes H$ are of the form $x \otimes 1$ and $1 \otimes y$ for $x$ and $y$ primitive).

If dealing with a non-zero characteristic base ring, we work from the Verschiebung. Take $x \in H$ such that $V^{r}(x)=0$ but $V^{r-1}(x)=b \neq 0$ (Recall we deal only with connected Hopf algebras). Pick a positive $m$ and $\hat{x} \in$ $\operatorname{Hom}_{\mathcal{H} \mathcal{A}}(H(m), H)$ given by $\hat{x}(1)=1, \hat{x}\left(\omega_{m}\right)=b$ and $\hat{x}\left(\omega_{i}\right)=0$ for $i \neq m$. As above, we define $\hat{x} \circ \hat{1}=\varphi^{m}(\hat{x}) \circ \varphi^{0}(\hat{1})=\varphi^{m}(\alpha)\left(\right.$ where $\left.\alpha \in \operatorname{Hom}_{\mathcal{H A}}(H(m), H)\right)$, and the rest follows.

\section{Cobilinear maps and coring structures on connected Hopf algebras}

Next we build for algebras a theory corresponding to what was done for coalgebras, defining cobilinear maps, universal cobilinear maps and the notion of a Hopf coring.

We start with Hopf algebras over a commutative ring $R$. Let $H_{1}, H_{2}$ and $K$ be such Hopf algebras. A morphism of algebras

$$
\phi: K \rightarrow H_{1} \otimes H_{2}
$$

is called a cobilinear map if the following diagrams commute:
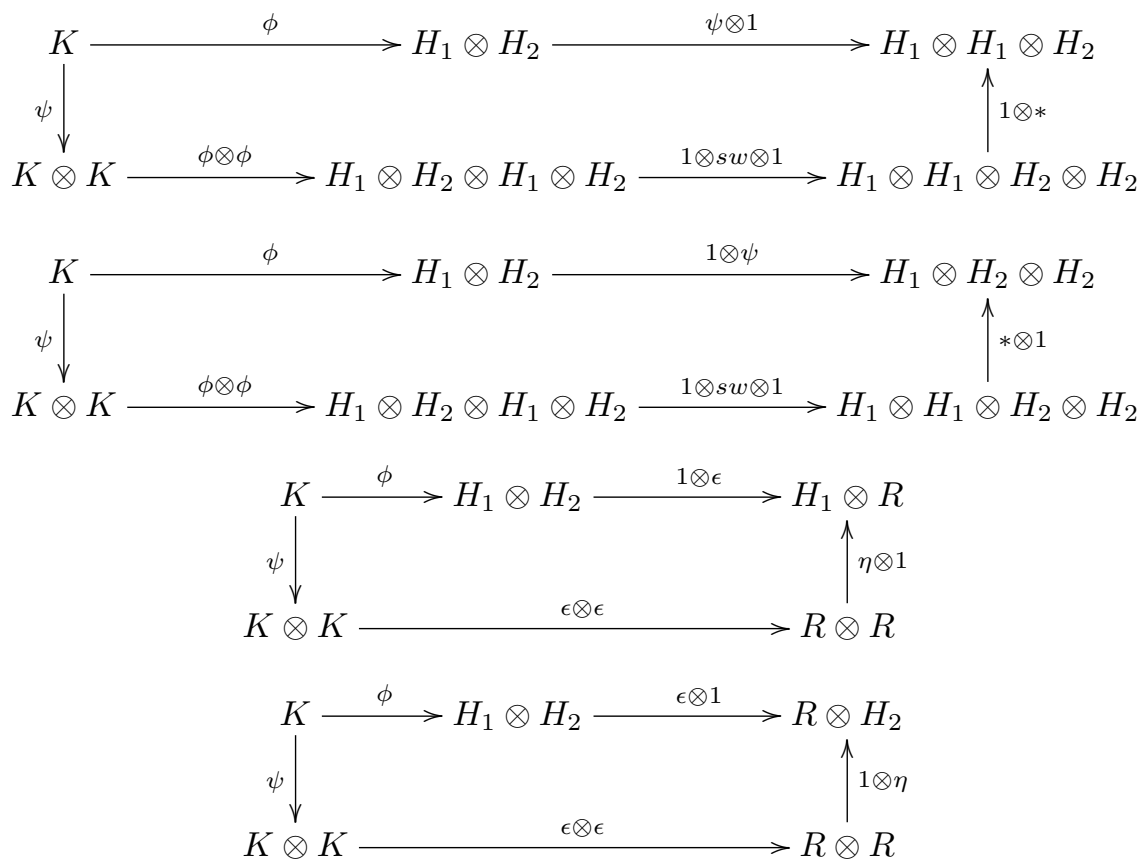
where in each case $\psi$ and $*$ stand respectively for Hopf algebra coproduct and product, $\eta$ and $\epsilon$ are respectively the unit and the counit, and $s w$ is an algebra switch map taking a $a \otimes b$ to $b \otimes a$.

The diagrams reflect the inversion of the diagrams one can construct to define the bilinearity of maps between coalgebras.

As for bilinear products, we can also describe cobilinear maps in terms of relations, but these don't come up as neatly as the above diagrams. Given $x \in K$ and $\phi: K \rightarrow H_{1} \otimes H_{2}$ cobilinear, denote $\phi(x)$ by $\sum x_{(1)} \otimes x_{(2)}$ (superscripts are reserved for coproducts). Using this notation, we then get, for any $x \in K$ :

$(1) \sum\left(x_{(1)}\right)^{(1)} \otimes\left(x_{(1)}\right)^{(2)} \otimes x_{(2)}=\sum\left(x^{(1)}\right)_{(1)} \otimes\left(x^{(2)}\right)_{(1)} \otimes\left[\left(x^{(1)}\right)_{(2)}\left(x^{(2)}\right)_{(2)}\right]$,

$(2) \sum x_{(1)} \otimes\left(x_{(2)}\right)^{(1)} \otimes\left(x_{(2)}\right)^{(2)}=\sum\left[\left(x^{(1)}\right)_{(1)}\left(x^{(2)}\right)_{(1)}\right] \otimes\left(x^{(1)}\right)_{(2)} \otimes\left(x^{(2)}\right)_{(2)}$,

(3) $\sum x_{(1)} \otimes \epsilon_{H_{2}}\left(x_{(2)}\right)=\sum \eta_{H_{1}}\left(\epsilon_{K}\left(x^{(1)}\right)\right) \otimes \epsilon_{K}\left(x^{(2)}\right)$,

(4) $\sum \epsilon_{H_{1}}\left(x_{(1)}\right) \otimes x_{(2)}=\sum \epsilon_{K}\left(x^{(1)}\right) \otimes \eta_{H_{2}}\left(\epsilon_{K}\left(x^{(2)}\right)\right)$.

The universal cobilinear coproduct is defined, for each pair of Hopf algebras $H_{1}$ and $H_{2}$, when it exists, as the unique Hopf algebra $H_{1} \widehat{\otimes} H_{2}$ together with a cobilinear map (of algebras)

$$
\gamma: H_{1} \widehat{\otimes} H_{2} \rightarrow H_{1} \otimes H_{2}
$$

that is universal with respect to all cobilinear maps.

Theorem 3.1 ([20]). Any two connected Hopf algebras over a perfect field have a universal cobilinear coproduct.

The definition of a universal cobilinear coproduct for connected Hopf algebras gives us a way to define what a connected Hopf coring should be. This is given as a connected Hopf algebra $H$ together with a map $\phi: H \rightarrow H \widehat{\otimes} H$ that is coassociative, that is, such that $(\phi \widehat{\otimes} 1) \phi=(1 \widehat{\otimes} \phi) \phi$ as maps from $H$ to $H \widehat{\otimes} H \widehat{\otimes} H$.

By the universal construction, this is equivalent to giving two maps of algebras (two "coproducts") $H \rightarrow H \otimes H$ that are related by a codistributivity (see $[20])$.

Given a connected Hopf coring, its map $\phi: H \rightarrow H \widehat{\otimes} H$, when composed with the defining map $\gamma: H \widehat{\otimes} H \rightarrow H \otimes H$, gives an algebra map $\tilde{\phi}: H \rightarrow$ $H \otimes H$.

This map $\tilde{\phi}$, together with the original coproduct $\psi$, satisfies a form of codistributivity. This comes from the inversion of the diagrams from Section 2 that deal with distributivity for Hopf rings. 
We get then:
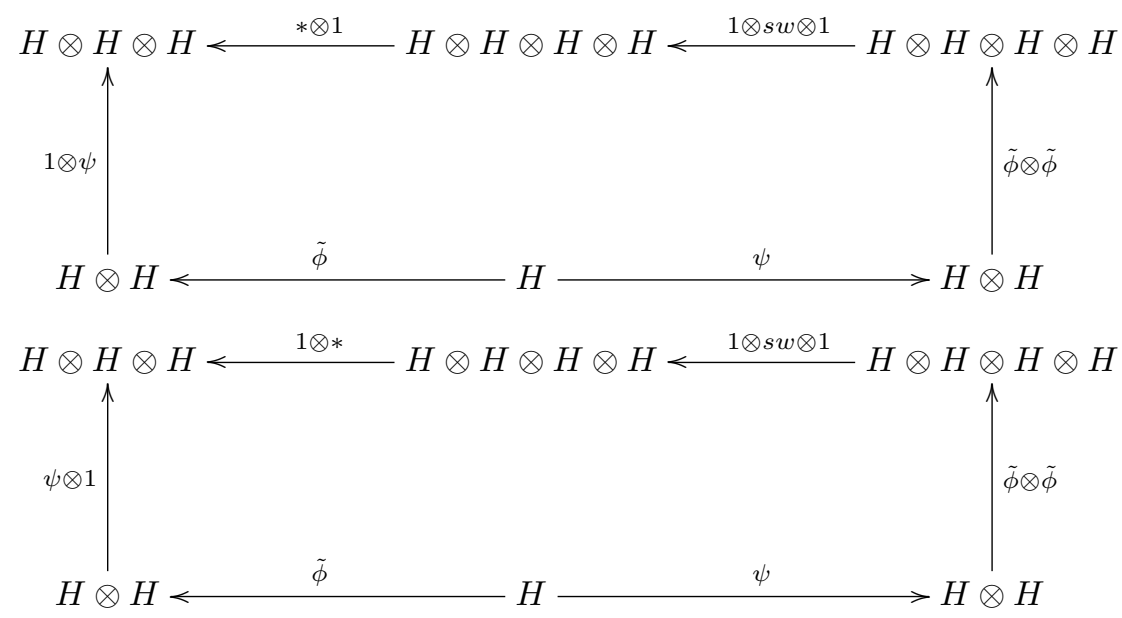

We can write the relations coming from these diagrams. Subscripts refer to the proper coproduct in each case, and for instance the first diagram yields:

$\sum x_{\tilde{\phi}}^{(1)} \otimes\left(x_{\tilde{\phi}}^{(2)}\right)_{\psi}^{(1)} \otimes\left(x_{\tilde{\phi}}^{(2)}\right)_{\psi}^{(2)}=\sum\left(x_{\psi}^{(1)}\right)_{\tilde{\phi}}^{(1)}\left(x_{\psi}^{(2)}\right)_{\tilde{\phi}}^{(1)} \otimes\left(x_{\psi}^{(1)}\right)_{\tilde{\phi}}^{(2)} \otimes\left(x_{\psi}^{(2)}\right)_{\tilde{\phi}}^{(2)}$ for each $x \in H$ (Compare equation (2) on page 632).

The following result states how cobilinear maps $\phi: K \rightarrow H_{1} \otimes H_{2}$ act on the primitives of $K$.

Lemma 3.2. Let $\phi: K \rightarrow H_{1} \otimes H_{2}$ be a cobilinear map. If $x \in K$ is a primitive element, then

$$
\phi(x)=\sum_{\alpha, \beta} p_{\alpha} \otimes p_{\beta},
$$

where all $p_{\alpha}$ and $p_{\beta}$ are primitive.

Proof. The first relation in the definition of cobilinear map (from page 631), whenever applied to a primitive $x$, gives

$$
\sum\left(x_{(1)}\right)^{(1)} \otimes\left(x_{(1)}\right)^{(2)} \otimes x_{(2)}=\sum\left[1 \otimes x_{(1)} \otimes x_{(2)}+x_{(1)} \otimes 1 \otimes x_{(2)}\right]
$$

and so $\psi\left(x_{(1)}\right)=1 \otimes x_{(1)}+x_{(1)} \otimes 1$ for any $x_{(1)}$.

The second relation from page 631 gives that any $x_{(2)}$ is also primitive.

\section{Induced Hopf ring structures}

Given a connected Hopf ring $H$ (with ring product $\circ$ ) and a coring structure on the Hopf algebra $\mathbf{F}_{p}\left[x_{0}, x_{1}, \ldots\right]$, we can use the Dieudonné equivalence on Hopf algebras to obtain a second, possibly different ring structure on the same Hopf algebra underlying $H$.

Let $\phi: \mathbf{F}_{p}\left[x_{0}, x_{1}, \ldots\right] \rightarrow \mathbf{F}_{p}\left[x_{0}, x_{1}, \ldots\right] \otimes \mathbf{F}_{p}\left[x_{0}, x_{1}, \ldots\right]$ be a cobilinear map that gives the Hopf algebra $\mathbf{F}_{p}\left[x_{0}, x_{1}, \ldots\right]$ its coring structure. From Lemma 
3.2 we know that it acts on the Witt polynomials by $\omega_{i} \mapsto \sum_{\alpha, \beta} \omega_{\alpha}^{i} \otimes \omega_{\beta}^{i}$, where all the $\omega_{\alpha}^{i}$ and $\omega_{\beta}^{i}$ are also Witt polynomials.

From this, we define a product $\mathrm{O}_{2}$ on the Dieudonné module $D H$ by writing, for $\varphi_{1}$ and $\varphi_{2}$ in $D H$,

$$
\varphi_{1} \circ_{2} \varphi_{2}\left(\omega_{i}\right)=\sum_{\alpha, \beta} \varphi_{1}\left(\omega_{\alpha}^{i}\right) \circ \varphi_{2}\left(\omega_{\beta}^{i}\right)
$$

if the result on the right is a sum of primitives of $H$, and zero otherwise.

This definition is represented by the following diagram:

$$
\mathbf{F}_{p}\left[x_{0}, x_{1}, \ldots\right] \stackrel{\phi}{\longrightarrow} \mathbf{F}_{p}\left[x_{0}, x_{1}, \ldots\right] \otimes \mathbf{F}_{p}\left[x_{0}, x_{1}, \ldots\right] \stackrel{\varphi_{1} \otimes \varphi_{2}}{\longrightarrow} H \otimes H \stackrel{\circ}{\longrightarrow} H
$$

From the end of Section 2, we know this induces a product $\mathrm{o}_{2}$ on $H$. If we have a characteristic zero ring, unraveling $\mathrm{O}_{2}$ gives

$$
x \circ_{2} 1=\hat{x} \circ_{2} \hat{1}\left(\omega_{m}\right)=\sum_{\alpha, \beta} \hat{x}\left(\omega_{\alpha}^{m}\right) \circ \hat{1}\left(\omega_{\beta}^{m}\right)=c x
$$

for some $c$ that depends on the cobilinear map inducing this product.

We can similarly define $1 \circ_{2} y$, and finally $x \circ_{2} y=\left(x \circ_{2} 1\right)\left(1 \circ_{2} y\right)$.

As before, for non-zero characteristic rings we work from the Verschiebung.

We get:

$$
x \circ_{2} 1=[\hat{x} \circ \hat{1}]\left(\omega_{m}^{r}\right)=\sum\left[\hat{x}\left(\omega_{\alpha}^{m}\right)\right]^{r} \circ\left[\hat{1}\left(\omega_{\beta}^{m}\right)\right]^{r}=c b^{r}
$$

for some $c$.

For general $x$ (with $V^{r}(x)=0$ but $V^{r-1}(x) \neq 0$ ) and $y$ (with $V^{s}(y)=0$ but $\left.V^{s-1}(y) \neq 0\right)$, define $\circ_{2}$ recursively on the primitive filtration by

$$
x \circ_{2} y=c\left[V^{r-1}(x)\right]^{r} \circ_{2}\left[V^{s-1}(y)\right]^{s},
$$

where again $c$ is a constant that depends on the coring structure put on $\mathbf{F}_{p}\left[x_{0}, x_{1}, \ldots\right]$.

We get thus the following result.

Proposition 4.1. Given a connected Hopf ring H, any coring structure on $\mathbf{F}_{p}\left[x_{0}, x_{1}, \ldots\right]$ induces a second Hopf ring structure on the Hopf algebra underlying $H$.

Note that, applying the equivalences from Section 2 between Hopf rings and their Dieudonné rings, one gets that this $\mathrm{o}_{2}$ on $H$ induces in its own right the same $\mathrm{O}_{2}$ on $\mathrm{DH}$ that was pictured above.

We will make explicit these induced products on $H$ for three specific coring structures on $\mathbf{F}_{p}\left[x_{0}, x_{1}, \ldots\right]$.

We start with the diagonal structure. This comes from the cobilinear map

$$
\mathbf{F}_{p}\left[x_{0}, x_{1}, \ldots\right] \rightarrow \mathbf{F}_{p}\left[x_{0}, x_{1}, \ldots\right] \otimes \mathbf{F}_{p}\left[x_{0}, x_{1}, \ldots\right]
$$

that attributes to each Witt polynomial $\omega_{i}$ the value $\omega_{i} \otimes \omega_{i}$.

This cobilinear map does determine a coring structure on the Witt Hopf algebra, since codistributivity from page 633 yields the following commutative 
diagrams for each Witt polynomial:

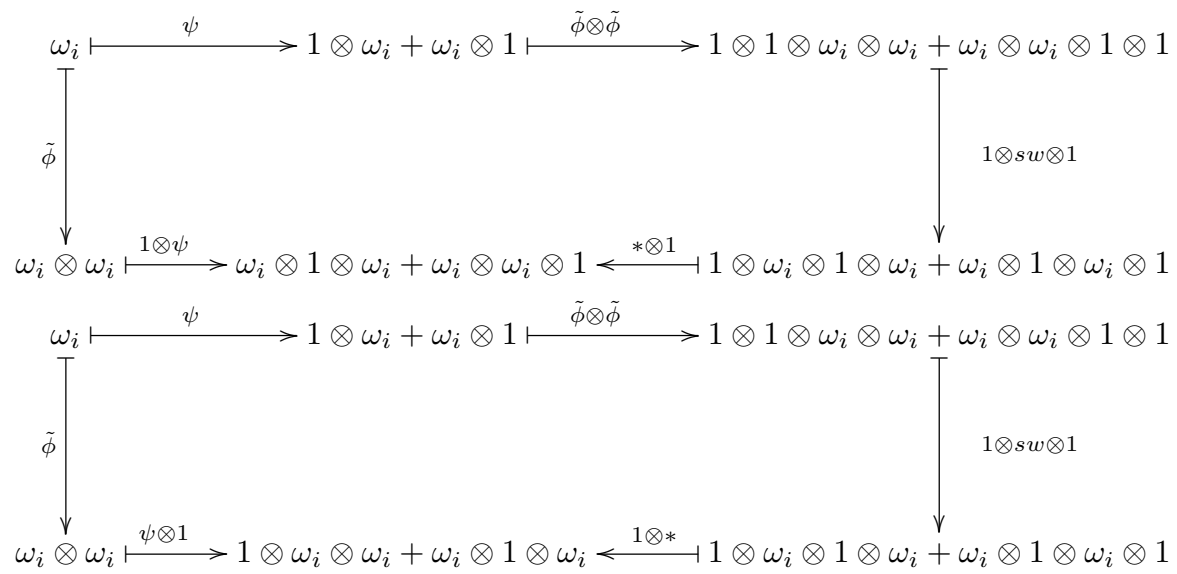

Determining the induced product on $H$, we get

$$
x \circ_{2} 1=[\hat{x} \circ \hat{1}]\left(\omega_{m}^{r}\right)=\sum\left[\hat{x}\left(\omega_{\alpha}^{m}\right)\right]^{r} \circ\left[\hat{1}\left(\omega_{\beta}^{m}\right)\right]^{r}=\left[\hat{x}\left(\omega_{m}\right)\right]^{r} \circ\left[\hat{1}\left(\omega_{m}\right)\right]^{r}=b^{r}
$$

(assuming as above that $V^{r-1}(x)=b \neq 0$ ) and

$$
x \circ_{2} y=\left[V^{r-1}(x)\right]^{r} \circ_{2}\left[V^{s-1}(y)\right]^{s} .
$$

Two other possible coring structures are the right $k$-shift (given by $\omega_{i} \mapsto$ $\omega_{i} \otimes \omega_{i+k}$ ) and the left $k$-shift (sending $\omega_{i}$ to $\omega_{i+k} \otimes \omega_{i}$ ).

Codistributivity for the right $k$-shift gives the following commutative diagrams (the left $k$-shift produces similar ones).
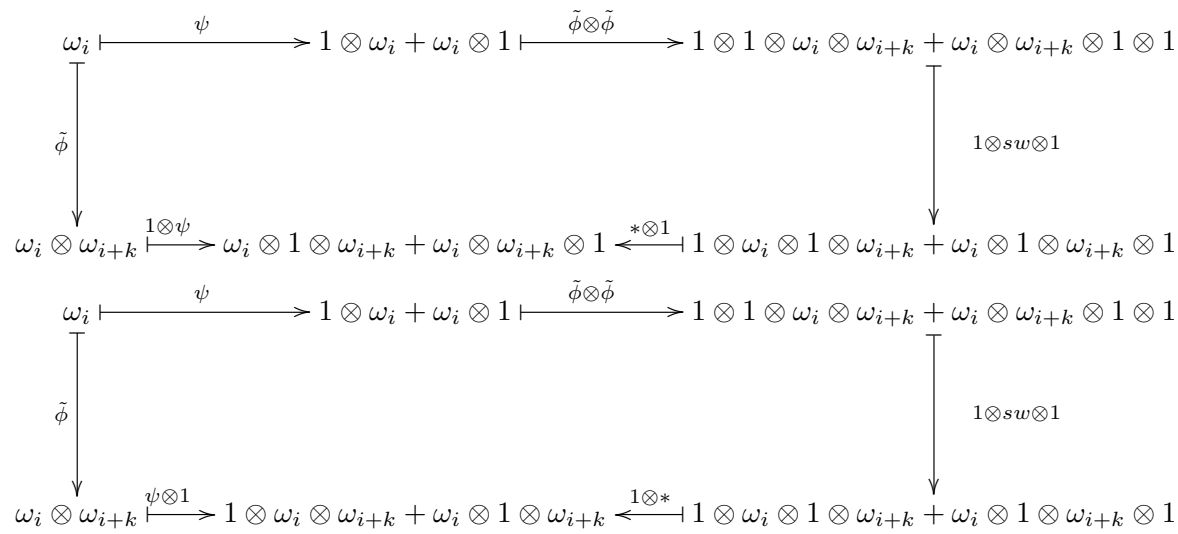

As these are not cocommutative coring structures, their induced ring products will not be commutative.

For the right $k$-shift, one gets

$$
x \circ_{2} 1=[\hat{x} \circ \hat{1}]\left(\omega_{m}^{r}\right)=\left[\hat{x}\left(\omega_{m}\right)\right]^{r} \circ\left[\hat{1}\left(\omega_{m+k}\right)\right]^{r}=b^{r}
$$


but

$$
1 \circ_{2} x=\left[\hat{1}\left(\omega_{m}\right)\right]^{r} \circ\left[\hat{x}\left(\omega_{m+k}\right)\right]^{r}=0
$$

Similarly, the left $k$-shift yields $x \circ_{2} 1=0$ and $1 \circ_{2} x=b^{r}$.

In a certain way, coring structures on $\mathbf{F}_{p}\left[x_{0}, x_{1}, \ldots\right]$ classify Hopf ring structures for a given connected Hopf algebra $H$ (We need the additional condition, though, that this Hopf algebra was indeed a Hopf ring, as that structure directly influences the construction of new Hopf ring structures on $H$ from coring structures on $\left.\mathbf{F}_{p}\left[x_{0}, x_{1}, \ldots\right]\right)$.

\section{The induced structures for connective Morava k-theory}

The induced structure development from the previous section can easily be applied to well-known settings, turning up new products for some important Hopf rings.

We will use the previous coring structures on $\mathbf{F}_{p}\left[x_{0}, x_{1}, \ldots\right]$ to define new products for Hopf rings coming from connective Morava $k$-theory (One can easily apply the same methods for complex cobordism Hopf rings), and see what the diagonal and $k$-shift structures can induce. Any other coring structure on $\mathbf{F}_{p}\left[x_{0}, x_{1}, \ldots\right]$ would naturally determine additional ring products.

Details on the Hopf rings that arise naturally from these theories can be found in [1], [12] and [22], and the determination of their corresponding Dieudonné rings are in [18]. These Dieudonné ring products are important in the determination of induced ring products for the underlying Hopf algebras.

For $n \geq 1, K(n)$ denotes the spectrum of the $2\left(p^{n}-1\right)$-periodic $n$-th Morava $k$-theory, and $k(n)$ the corresponding spectrum for connective theory. $p$ is a fixed odd prime.

Let $K(n)$ and $k(n)$ be the $\Omega$-spectra for the spectra $K(n)$ and $k(n)$. For each $r$, both $K \overline{(n)_{*}} \underline{K}^{*}(n), r$ and $K(n)_{*} \underline{k(n)}_{r}$ are Hopf algebras and both $K(n)_{*} K(n)$ and $K\left(n{\overline{k_{*}(n)}}^{r}\right.$ are Hopf rings.

$K(n)_{*} K(n)$ is generated by elements

$$
\begin{aligned}
& e_{1} \in K(n)_{1} \frac{K(n)}{1}, a_{(i)} \in K(n)_{2 p^{i}} \frac{K(n)}{1}(\text { for } i<n), \\
& b_{(i)} \in K(n)_{2 p^{i}} \frac{K(n)}{2},\left[v_{n}\right] \in K(n)_{0} \underline{K(n)}-2\left(p^{n}-1\right), \text { and also } \\
& {\left[v_{n}^{-1}\right] \in K(n)_{0} \frac{K(n)}{2\left(p^{n}-1\right)} .}
\end{aligned}
$$

We have that $K(n)_{*} \frac{k(n)}{K}$ is a sub-Hopf ring of $K(n)_{*} K(n)$ (The element $\left[v_{n}^{-1}\right]$ is not defined in $\left.\overline{K(n)} * k(n){ }_{*}\right)$. The $b_{i}$ come from the complex orientation of the Morava K-theories. The $a_{i}$ are standard homology elements.

Proposition 5.1 ([22], [1]). Let $p$ be an odd prime and $n \geq 1$. Then there exist elements $e_{1} \in K(n)_{1} \underline{k(n)}{ }_{1}, a_{i} \in K(n)_{2 i} \underline{k(n)}{ }_{1}\left(\right.$ for $\left.i<p^{n}\right), b_{i} \in$ $K(n)_{2 i} \frac{k(n)}{2}$. Let $a_{(i)}=a_{p^{i}}$ and $b_{(i)}=b_{p^{i}} . \psi$ denotes the coproduct, $*$ the algebra (or group) product and o the ring product. We have

i) $e_{1} \circ(-)$ is the homology suspension map. 
ii) $\psi\left(a_{i}\right)=\sum_{j=0}^{i} a_{i-j} \otimes a_{j}$ and $\psi\left(b_{i}\right)=\sum_{j=0}^{i} b_{i-j} \otimes b_{j}$.

iii) The standard mod $p$ homology $a_{i}$ and $b_{i}$ are all permanent cycles in the Atiyah-Hirzebruch spectral sequence for $K(n)_{*} k(n){ }_{*}$ and represent the corresponding $a_{i}$ and $b_{i}$.

iv) $e_{1} \circ e_{1}=-b_{(0)}$.

v) $a_{(i)} \circ a_{(j)}=-a_{(j)} \circ a_{(i)}$.

vi) $b_{(i)}^{* p}=0$.

vii) $a_{(i)}^{* p}=0$ for $i<n-1$.

viii) $a_{(n-1)}^{* p}=v_{n} a_{(0)}-a_{(0)} \circ b_{(0)}^{\circ\left(p^{n}-1\right)} \circ\left[v_{n}\right]$

ix) $v_{n} e_{1}=b_{(0)}^{\circ\left(p^{n}-1\right)} \circ e_{1} \circ\left[v_{n}\right]$.

x) $b_{(k)}^{\circ p^{n}} \circ\left[v_{n}\right]=v_{n}^{p^{k}} b_{(k)}$ for $k \geq 0$.

We will restrict our attention to the reduced Morava k-theories $\overline{K(n)}_{*}(-)$ one gets by setting $v_{n}=1$.

The Hopf algebras $K(n) * \underline{k(n)} k$ are not connected, so we are not able to get the induced products directly as in the previous sections (the element [1] does not have eventually trivial Verschiebung). Consider then just its connected part.

We get, for each generator,

$V\left(a_{(0)}\right)=0$,

$V\left(a_{(i+1)}\right)=a_{(i)}$ for $i=0, \ldots, n$

$V\left(b_{(0)}\right)=0$,

$V\left(b_{(k+1)}\right)=b_{(k)}$ for $k \geq 0$,

$V\left(e_{1}\right)=0$,

and so we can easily get the induced products for the structures exemplified in the previous section.

For the diagonal structure, we obtain:

$$
\begin{aligned}
& a_{(i)} \circ_{2} a_{(j)}=\left[V^{i-1}\left(a_{(i)}\right)\right]^{i}\left[V^{j-1}\left(a_{(j)}\right)\right]^{j}=a_{(0)}^{i} a_{(0)}^{j}=a_{(j)} \circ_{2} a_{(i)}, \\
& a_{(i)} \circ_{2} b_{(k)}=\left[V^{i-1}\left(a_{(i)}\right)\right]^{i}\left[V^{k-1}\left(b_{(k)}\right)\right]^{k}=a_{(0)}^{i} b_{(0)}^{k}=b_{(k)} \circ_{2} a_{(i)}, \\
& b_{(k)} \circ_{2} b_{(m)}=\left[V^{k-1}\left(b_{(k)}\right)\right]^{k}\left[V^{m-1}\left(b_{(m)}\right)\right]^{m}=b_{(0)}^{k} b_{(0)}^{m}=b_{(m)} \circ_{2} b_{(k)} .
\end{aligned}
$$

The right $k$-shift gives

$$
\begin{aligned}
& a_{(i)} \circ_{2} 1=a_{(0)}^{i}, \\
& b_{(k)} \circ_{2} 1=b_{(0)}^{k},
\end{aligned}
$$

and all other products zero.

Finally, the left $k$-shift yields a symmetric set of relations:

$$
\begin{aligned}
& 1 \circ_{2} a_{(i)}=a_{(0)}^{i}, \\
& 1 \circ_{2} b_{(k)}=b_{(0)}^{k}
\end{aligned}
$$

with, again, all other products zero. 


\section{References}

[1] J. M. Boardman, R. L. Kramer, and W. S. Wilson, The periodic Hopf ring of connective Morava K-theory, Forum Math. 11 (1999), no. 6, 761-767.

[2] A. K. Bousfield, On $\lambda$-rings and the $K$-theory of infinite loop spaces, K-Theory 10 (1996), no. 1, 1-30.

3] On p-adic $\lambda$-rings and the K-theory of $H$-spaces, Math. Z. 223 (1996), no. 3 , 483-519.

[4] V. Buchstaber and A. Lazarev, Dieudonné modules and p-divisible groups associated with Morava K-theory of Eilenberg-Mac Lane spaces, Algebr. Geom. Topol. 7 (2007), $529-564$.

[5] M. Demazure, Lectures on p-Divisible Groups, Lecture Notes in Mathematics, Vol. 302. Springer-Verlag, Berlin-New York, 1972.

[6] M. Demazure and P. Gabriel, Groupes Algébriques, North-Holland, Amsterdam, 1970.

[7] P. G. Goerss, Hopf rings, Dieudonné modules, and $E_{*} \Omega^{2} S^{3}$, Homotopy invariant algebraic structures (Baltimore, MD, 1998), 115-174, Contemp. Math., 239, Amer. Math. Soc., Providence, RI, 1999.

[8] P. Goerss, J. Lannes, and F. Morel, Hopf algebras, Witt vectors, and Brown-Gitler spectra, Algebraic topology (Oaxtepec, 1991), 111-128, Contemp. Math., 146, Amer. Math. Soc., Providence, RI, 1993.

[9] M. J. Hopkins, D. C. Ravenel, and W. S. Wilson, Morava Hopf algebras and spaces $K(n)$ equivalent to finite Postnikov systems, Stable and unstable homotopy (Toronto, ON, 1996), 137-163, Fields Inst. Commun., 19, Amer. Math. Soc., Providence, RI, 1998.

[10] J. R. Hunton and P. R. Turner, Coalgebraic algebra, J. Pure Appl. Algebra 129 (1998), no. 3, 297-313.

[11] I. Kaplansky, Bialgebras, Chicago, 1975

[12] R. L. Kramer, The periodic Hopf ring of connective Morava K-theory, Ph. D. Thesis, The Johns Hopkins University, 1990.

[13] J. W. Milnor and J. C. Moore, On the structure of Hopf algebras, Ann. of Math. (2) 81 (1965), 211-264.

[14] D. C. Ravenel, Dieudon'e modules for abelian Hopf algebras, Conference on homotopy theory (Evanston, Ill., 1974), 177-183, Notas Mat. Simpos., 1, Soc. Mat. Mexicana, México, 1975.

[15] D. C. Ravenel and W. S. Wilson, The Hopf ring for complex cobordism, J. Pure Appl. Algebra 9 (1976/77), no. 3, 241-280.

[16] _ The Morava K-theories of Eilenberg-Mac Lane spaces and the Conner-Floyd conjecture, Amer. J. Math. 102 (1980), no. 4, 691-748.

[17] H. Sadofsky and W. S. Wilson, Commutative Morava homology Hopf algebras, Homotopy theory via algebraic geometry and group representations (Evanston, IL, 1997), 367-373, Contemp. Math., 220, Amer. Math. Soc., Providence, RI, 1998.

[18] R. M. Saramago, Dieudonné rings associated with $K(n)_{*} \underline{k(n)} *$, Proc. Amer. Math. Soc. 136 (2008), no. 8, 2699-2709.

[19] _ Dieudonné module structures for ungraded and periodically graded Hopf rings, Algebras and Representation Theory 13 (2010), no. 5, 521-541.

[20] _ Connected Hopf Corings and Their Dieudonné Counterparts, preprint.

[21] C. Schoeller, Étude de la catégorie des algèbres de Hopf commutatives connexes sur un corps, Manuscripta Math. 3 (1970), 133-155.

[22] W. S. Wilson, The Hopf ring for Morava K-theory, Publ. Res. Inst. Math. Sci. 20 (1984), no. 5, 1025-1036. 
Departamento de Matemática

Instituto Superior TÉCNico

Av. Rovisco Pais, 1049-001 Lisboa, Portugal

E-mail address: saramago@math.ist.utl.pt 